\title{
Margot IRVINE
}

\section{Une réévaluation $\mathrm{du}$ « $\mathrm{mal}$ du siècle »}

Avec une série d'articles parus dans les années soixante-dix ${ }^{1}$ et deux livres publiés en 1977 et $1987^{2}$, Chantal Bertrand-Jennings a été l'une des premières critiques à analyser la représentation de la femme dans la fiction d'auteurs réalistes et naturalistes. Elle s'est ensuite tournée vers les ouvrages des romancières, surtout celles qui ont écrit pendant la première moitié du XIX ${ }^{\mathrm{e}}$ siècle. Le présent ouvrage approfondit et affine ce travail, et ses conclusions sont importantes. Un courant essentiel de la critique féministe contemporaine vise à présenter une histoire littéraire qui tienne compte de la production littéraire des femmes. La contribution des auteures de la période romantique est mise en évidence dans Un autre mal $d u$ siècle. En réintégrant des romans d'auteures tout en prenant en considération les notions clés du romantisme, cet ouvrage nuance la façon dont on perçoit le «mal du siècle» et transforme notre compréhension des grands mouvements littéraires du XIX ${ }^{\mathrm{e}}$ siècle. C'est un modèle des réévaluations dont une critique féministe est capable.

Le « mal du siècle », ce vague malaise qui, ressenti par plusieurs auteurs de la période romantique, implique un manque de volonté, une mélancolie introspective, un culte du moi, une complaisance dans la souffrance et un repli sur soi (p. 10), Bertrand-Jennings le trouve aussi dans plusieurs œuvres de femmes, œuvres qui n'ont pas été prises en compte lors de la théorisation du concept. Toutefois, à la différence de

\footnotetext{
${ }^{1}$ Voir, entre autres, Chantal Jennings, «La Dualité de Maupassant : son attitude envers la femme », Revue des Sciences Humaines, tome XXXV, $\mathrm{n}^{\circ} 140$, oct-déc. 1970, p. 559-578; «Perspective féministe sur un "romancier pour dames": Alphonse Daudet», La Pensée, no 170, août 1973, p. 86-106; «Zola féministe? I », Cahiers naturalistes, $\mathrm{n}^{\circ} 44$, 1972, p. 172-187; «Zola féministe? II », Cahiers naturalistes, ${ }^{\circ} 45,1973$, p. 86-106.

${ }^{2}$ Id., L'Éros et la femme chez Zola: de la chute au paradis retrouvé, Paris, Klincksieck, 1977, et Espaces romanesques : Zola, Sherbrooke, Naaman, 1987.
} 
ses homologues masculins, Bertrand-Jennings constate que, dans les textes de femmes, l'exclusion dont souffre le ou la protagoniste a toujours une source concrète. Partant de la supposition que l'inscription de l'expérience du vécu des femmes, transposée dans le champ du symbolique, peut se lire aux niveaux diégétique, langagier et symbolique des textes - supposition que les romans de femmes semblent confirmer - , Bertrand-Jennings examine la problématique de l'exclusion dans les textes de femmes de l'époque (p. 21).

Dans le chapitre premier, elle effectue une lecture serrée des deux grands romans de Germaine de Staël, Delphine (1802) et Corinne, ou l'Italie (1807). Dans Delphine, plusieurs représentations d'exclusion sociale et d'aliénation spécifiquement féminines sont relevées, tandis que dans Corinne, une forme d'exclusion sociale est privilégiée : celle de la femme d'exception. Ainsi, comme le montre Bertrand-Jennings, il faudrait considérer que c'est Staël plutôt qu'Alfred de Vigny (Chatterton, 1835) qui inaugure le thème romantique du malheur du génie incompris. Bertrand-Jennings montre ensuite que, comme dans les ouvrages de Germaine de Staël, le souci social et un intérêt pour la représentation des conditions de vie des femmes se trouvent dans les textes de plusieurs romancières en vogue dans la première moitié du XIX ${ }^{\mathrm{e}}$ siècle. À partir d'un choix d'ouvrages de Stéphanie de Genlis (Mademoiselle de Clermont, 1802), Sophie Cottin (Amélie Mansfield, 1803), Julie de Krüdener (Valérie, 1804), Sophie Gay (Anatole, 1815), Hortense Allart de Méritens (Gertrude, 1828) et Daniel Stern (pseudonyme de Marie d'Agoult, Nélida, 1846), Bertrand-Jennings relève les figures d'exclues (étrangères, infirmes, filles-mères, femmes du peuple) qui abondent dans ces romans. Le souci social que l'examen de ces figures implique de la part des romancières préfigure le roman réaliste et naturaliste, et nous force à modifier la périodicité de notions telles que le « romantisme social », que l'on date habituellement seulement des années 1840. La multiplicité des figures d'exclues rend la démonstration très convaincante. Plusieurs des romancières étudiées dans ce livre (Germaine de Staël, George Sand, Flora Tristan, Claire de Duras, Marceline Desbordes-Valmore) ont bénéficié au cours de ces dernières années de nombreuses études critiques et de rééditions de leurs ouvrages, mais ce chapitre indique qu'il reste à explorer de nombreux romans de femmes de la période romantique et il donne envie de mieux les connaitre. 
Bertrand-Jennings sait dénicher des textes intéressants. La redécouverte des œuvres de Claire de Duras est due en grande partie à ses articles $^{3}$ et à son livre D'un siècle l'autre : romans de Claire de Duras (2001). En examinant dans le présent ouvrage les trois romans publiés du vivant de Claire de Duras (Ourika, Édonard, Olivier), elle montre l'extraordinaire sensibilité au sort de l'exclu(e) dont fait preuve cette auteure. Ici encore, le mal du siècle dont souffrent les personnages durassiens est dû à des facteurs bien concrets : la peau noire d'Ourika, la classe sociale d'Édouard, et le mal peut-être physiologique, mais qu'on ne nomme pas, d'Olivier. Non seulement ces facteurs figurent l'exclusion des femmes, mais la façon dont chacun des personnages en arrive à collaborer à son propre assujettissement révèle l'impuissance, voire l'aliénation des femmes de l'époque romantique.

Bertrand-Jennings montre également que, comme les personnages durassiens, les personnages de fiction de Marceline Desbordes-Valmore aspirent à une intégration sociale qui leur est impossible. Dans les romans et nouvelles L'Atelier d'un peintre (1833), Contes (1840), Domenica (1843), Huit Femmes (1845) et Les petits Flamands $(1868)^{4}$, nous trouvons des personnages exclus de la société en raison de leur niveau social (pauvreté), de leur différence physique (infirmité, race), de leur âge (enfants) ou de leur métier (comédiens ou artistes). Bertrand-Jennings montre que, par son engagement social, la fiction de DesbordesValmore préfigure «à la fois le romantisme social à la Hugo ou même parfois l'enquête sociologique naturaliste» (p. 86). Elle y distingue aussi une vocation à tendance messianique, qu'elle décèle également dans le récit de voyage de Flora Tristan, Les Pérégrinations d'une paria (1833-1834). Effectivement, un des grands mérites de l'ouvrage de Bertrand-Jennings

\footnotetext{
${ }^{3}$ Id., «Condition féminine et impuissance sociale; les romans de la duchesse de Duras », Romantisme, n 63, 1989, p. 39-50; «Problématique d'un sujet féminin en régime patriarcal : Ourika de Mme de Duras », Nineteenth Century French Studies, Vol. 23, No. 1-2 (Fall-Winter 1994-1995), p. 42-58; «Vers un nouveau héros: Édouard de Claire de Duras ", French Review, Vol. 68, No. 3, 1995, p. 445-456; «Codes de l'honneur dans Olivier ou le secret de Claire de Duras », dans Chantal Bertrand-Jennings (dir.), Masculin / Féminin: le dix-neuvième siècle à l'épreuve du genre, Toronto, Centre d'études du $\mathrm{XIX}^{\mathrm{e}}$ siècle Joseph Sablé, 1999, p. 89-104.

${ }^{4}$ Tous ces textes ont été réédités dans les années 1990 par Marc Bertrand.
} 
est qu'il nous invite à faire d'intéressantes comparaisons entre les œuvres de femmes qu'il présente.

Se nommant "paria», terme utilisé auparavant par Germaine de Staël dans De la littérature (1800) pour désigner la femme de lettres, Flora Tristan désigne l'exclusion sociale qu'elle ressent en tant que femme du peuple, séparée de son mari. Dans son récit, elle fait preuve d'une grande solidarité avec les autres exclus qu'elle rencontre au cours de son voyage : matelots exploités, esclaves, Indiens, jeunes filles et femmes de toutes les conditions sociales. L'exploration sociale que fait Tristan dans ce récit de voyage peut aussi être considérée comme une forme d'enquête sociologique naturaliste. Les textes qu'elle écrit de retour en France poursuivront dans cette veine. Selon Bertrand-Jennings, le cheminement existentiel et littéraire de Tristan représente de façon exemplaire la condition féminine de la première moitié du XIX siècle (p. 107).

Dans le dernier chapitre de son ouvrage, Bertrand-Jennings examine deux romans de George Sand : Lélia et Gabriel. Ce qui intéresse dans Lélia est la réécriture que, de la première version du roman (1833) à la seconde (1839), Sand fait du « mal du siècle ». Tandis que la Lélia de la première version souffre d'un mal indéfini, Bertrand-Jennings montre que, dans la version de 1839, "l'héroïne guérit de son "mal" et Trenmor la convertit à l'action et à l'engagement dans la société » (p. 115). Le roman rejoint les autres textes de femmes étudiés dans ce volume par sa défense des exclus (le proscrit Valmarina-Trenmor, la femme forte et intelligente qu'est Lélia), par sa prise de position en faveur d'une société plus équitable et par son messianisme. Il critique toutefois certaines positions adoptées dans les autres ouvrages de femmes. Ainsi, Lélia refuse de se sacrifier à l'amour comme le font les héroïnes de Delphine ou d'Olivier. De plus, les caractéristiques sociosexuelles stéréotypées, adoptées par plusieurs héros et héroïnes des autres romans d'auteures, sont ici mises en question. Lélia revêt des caractéristiques viriles tandis que, comme le montre BertrandJennings, Sténio est fortement féminisé (p. 116). Cette réflexion sur la question du genre est reprise et approfondie par Sand dans son roman dialogué Gabriel, qui date de la même année que la deuxième version de Lélia. Gabriel/le est un personnage androgyne, motif qui renvoie à 
de nombreux textes de l'époque (Mademoiselle de Maupin, Fragoletta et Séraphîta, par exemple), à la différence que la portée idéologique du roman de Sand est clairement féministe. La tragédie de Gabriel/le est que le personnage ne peut pas combiner le rôle masculin qu'on lui a appris avec son sexe anatomique, ni «concilier son épanouissement affectif et intellectuel avec son exigence morale de dignité humaine » (p. 126). Sand reconceptualise ici l'opposition binaire masculin / féminin telle qu'elle a été construite, ainsi que la notion d'une identité sexuelle stable, et Bertrand-Jennings fait ressortir la modernité saisissante de ce questionnement.

Si l'œuvre de Sand est exceptionnelle à bien des égards, elle rejoint néanmoins celle des autres femmes de lettres de la période romantique $\mathrm{du}$ fait de sa fascination pour le personnage de l'exclu. Loin de se complaire dans leur souffrance et de revendiquer leur unicité comme les héros des textes masculins canoniques, les protagonistes des textes de femmes aspirent à une intégration sociale et s'appliquent souvent avec énergie et élan à la rechercher. Elles ne valorisent pas leur différence comme les héros du «mal du siècle », mais cette différence les rend sensibles aux autres victimes de la société. La compassion mène souvent, dans ces œuvres, à un intérêt prononcé pour les questions sociales. Bertrand-Jennings nous convainc ainsi qu'il faudrait citer ces romancières parmi les précurseurs du réalisme et du naturalisme en France.

Ce livre sera désormais incontournable pour les spécialistes de la littérature des femmes au XIX ${ }^{\mathrm{e}}$ siècle et sera lu avec profit par tous ceux qui s'intéressent à la littérature romantique. Il illustre également l'importance d'intégrer les textes de femmes à toute étude de mouvement littéraire afin d'en donner une compréhension plus juste et tout à la fois plus nuancée.

Référence : Chantal Bertrand-Jennings, Un autre mal du siècle : le romantisme des romancières, 1800-1846, Toulouse, Presses Universitaires du Mirail, 2005, 167 p. 